\title{
Effect of Solvent Content on the Properties of Nanostructure Silica Thin Film by Sol-Gel
}

\author{
A. Shokuhfar, E. Eghdam*, M. Alzamani \\ Mechanical Department (Advanced Materials and Nanotechnology Research Lab), K.N. Toosi University of Technology, Tehran, 19697 \\ 64499, Iran
}

\begin{abstract}
It is known that silica sol-gel coatings microstructure depend on the components of the sol. In order to develop different microstructures of the silica coatings and comparing their optical properties, a variety of silica sols were prepared which were consist of TEOS, water, catalyst and changing the solvent content, at room temperature. Prepared glass surfaces were coated with silica sol using a dipping technique, with constant withdrawing rate. Prepared coatings were investigated by field-emission scanning electron microscopy (FE-SEM) and UV-Vis. spectroscopy. Transmittance spectra showed that silica coatings featured a distinct increase of substrate transmittance at all wavelengths (400-1100 nm). The result indicated that by increasing the solvent content, the porosity of coating increased and the coating was more densified therefore the silica coating transmittance increased by $3-4 \%$ points. FE-SEM showed that the nanostructure silica coating grain size were estimated $\sim 20-40 \mathrm{~nm}$.
\end{abstract}

Keywords Solvent content, Silica, Transmittance, Sol-gel, Dip-coating

\section{Introduction}

$\mathrm{SiO}_{2}$ thin films have been widely used for optical coatings as anti-reflective and protective layers[1-3] and also for different microelectronic structures, as diffusion barriers and/or insulating layers with additional planarization ability[4-7]. Recently, the physico-chemical properties of $\mathrm{SiO}_{2}$ thin films have being applied in various methods to improve photovoltaic devices, as encapsulant coatings and/or to isolate electrical contacts in the cell[8-10].

To prevent disturbances of external light and increase the transmittance of incident light, antireflective technologies are widely applied in optical components, solar cells, displays, automotive glass, thermochromic windows, etc[11].

The sol-gel process is one of the most important techniques to coat glasses, ceramics and metals with amorphous thin films. There is great technological interest in the preparation of colored and multilayered dielectric films by sol-gel [12]. Researches and applications for the coating from sol have recently been reviewed by Schroeder[13], Dislich and Hussman[14], Dislich[15], Sakka[16] and Orgaz et al.[17].

Sol-gel technology can produce vitreous and ceramic coatings with various compositions over different substrates with low cost. Sol-gel $\mathrm{SiO}_{2}$ coatings are obtained by spining,

* Corresponding author:

ebrahim.eghdam@yahoo.com(E. Eghdam)

Published online at http://journal.sapub.org/nn

Copyright (C) 2012 Scientific \& Academic Publishing. All Rights Reserved dipping or spraying a solution prepared by dissolving aprecursor alkoxide in alcohol, catalyzed in an acid and hydrolyzed with water. After the deposition process, densification of the layers is obtained by heating at moderate temperatures $\left(300-500^{\circ} \mathrm{C}\right)$ in furnace or inert atmosphere. The resulting sol-gel $\mathrm{SiO}_{2}$ coatings have shown excellent mechanical and chemical stability, with optical and electrical properties that can be controlled by the sol preparation parameters[2,4,6].

In order to shift the reflectance value to minimum, a fine control of the coating thickness and refractive index is required[18,19]. Nearly zero-reflectance can be reached if "Eq.(1)" was established

$$
n_{c}=\left(n_{0} n_{s}\right)^{1 / 2}
$$

Where $n_{c}, n_{o}$ and $n_{s}$ are refractive indices of the coating,incident medium and substrate, respectively. According to Eq. 1 in the air atmosphere, considering refractive indices of interesting glazing materials for solar energy applications as 1.47-1.57, sol-gel anti-reflective coatings must present index values within the range of 1.21-1.25, which is lower than that of any known solid material. Nonetheless, as the refractive index of a material is related to its density, by increasing the porosity, its value can be dramatically reduced. The relationship between refractive index and porosity is depicted by the "Eq.(2)"

$$
n_{p c}=\left[(1-p / 100)\left(n_{d c}^{2}-1\right)+1\right]^{1 / 2}
$$

Where $\mathrm{n}_{\mathrm{pc}}, \mathrm{n}_{\mathrm{dc}}$ and $\mathrm{P}$ are the refractive indices of porous and densified coatings, and the porosity percentage, respectively[20]. 


\section{Experimental}

\subsection{Sample preparation}

All chemicals were used as received without further purification. The anti-reflective films were deposited on $2 \mathrm{~mm}$ thick automotive glass slides $\left(100 \times 40 \mathrm{~mm}^{2}\right)$ by dip-coating method. Withdrawal rate was adjusted to $1 \mathrm{~cm} / \mathrm{min}$. Anti-reflective sols were prepared by mixing absolute ethanol (EtOH, Merck, min 96 vol. \%), distilled water and tetraethyl orthosilicate (TEOS, Acros Organics, 98\%) according to the flow chart presented in Figure 1. The ethanol/TEOS molar ratios were 10, 15, 20 and 25 and the water/TEOS molar ratio was 2 in order to fit the thickness of the coatings to the maximum transmittance.

To prepare the suspensions, TEOS was mixed with one-half of ethanol using a magnetic stirrer for $15 \mathrm{~min}$. In a separate vessel, the remained ethanol was dissolved into distilled water and then the distilled water-ethanol mixture was added to TEOS-ethanol suspension drop by drop. When the mixture was homogeneous, $\mathrm{HCl}$ (Scharlau, 37\%) was added drop by drop until the $\mathrm{pH}$ of the solution decrease to the value of 2 . The anti-reflective sols were aged for $24 \mathrm{~h}$ before proceeding to coat. All the coatings were dried in air at room temperature for $24 \mathrm{~h}$ and subsequently were heated up to $500^{\circ} \mathrm{C}$ with the rate of $10^{\circ} \mathrm{C} / \mathrm{minfor} 30 \mathrm{~min}$.

\subsection{Sample characterization}

UV-Vis transmittance measurements on coating were performed using a Perkin Elmer UV/Vis Spectrophotometer, Lambda 25 tescan model vega-Ii. The size of $\mathrm{SiO}_{2}$ particles in coatings and surface morphology were investigated using a S-4160 Field Emission Scanning Electron Microscopy (FE-SEM) operating at $15 \mathrm{kV}$.

\section{Results and Discussion}

In general, many interdependent factors can play a prominent role in the final physical properties of sol-gel coatings: (i) sol-gel solution (ingredients, sequence of mixing, concentration and ratio of components, temperature, $\mathrm{pH}$ ); (ii) applying technique (dip, spin, spray, laminar flow); (iii) substrate (glasses, plastics, metals, ceramics) and its surface preparation; (iv) coating (porosity, residual $\mathrm{OH}$, structure, roughness, thickness). Dip-coating is the most popular method in which the colloids are evaporation induced and self-assembled on the substrate as it slowly withdrawn from the colloidal suspension. In this study several different silica sols were prepared to find the optimal deposition conditions on the microscope glass substrates for anti-reflective behavior.

The thickness of the porous $\mathrm{SiO}_{2}$ and therefore, the wavelength of the maximum transmittance, were finely tuned by controlling solvent ratio in the sol preparation stage .In Figure 2, substrate transmittance spectra (bare sample) and coated samples with nanostructure $\mathrm{SiO}_{2}$ (as-received) with different ratio of precursor dilution factor
$(\mathrm{D}=[\mathrm{EtOH}] /[\mathrm{TEOS}])$ is illustrated, which all were coated at the rate of $1 \mathrm{~cm} / \mathrm{min}$. As one can deduce, $\mathrm{SiO}_{2}$ nanostructure coating on the glass substrate, increase the transmittance percentage in the UV-Vis test. Considering figure 2, transmittance spectra at wavelength of $550 \mathrm{~nm}$ is maximum and around $2.3 \%$ of increment in the transmittance relative to the bare sample achieves when using $\mathrm{D}=10$ (AR-10 sample) in the solution. By increasing the $\mathrm{D}$ ratio from 10 to 15 , the maximum value and its transmittance percentage shift to the wavelength of $500 \mathrm{~nm}$ and $92.9 \%$ respectively (AR-15 sample). In this case, before and after the maximum point in the specified wavelength, transmittance spectra increase and decreased, respectively.

By increasing the D ratio from 15 to 20 (AR-15 to AR-20) the maximum transmittance didn't amplitude the amount of spectra, whereas the maximum transmittance is preserve approximately at the $500 \mathrm{~nm}$. According to Figure 2, increasing the $\mathrm{D}$ ratio more than 20 reduced the maximum value, obviously.Of course, one can observe that the sample which was coated in the sol with $\mathrm{D}=25$ shown lower transmittance compared to the sample which was coated in the sol with lower D ratio.

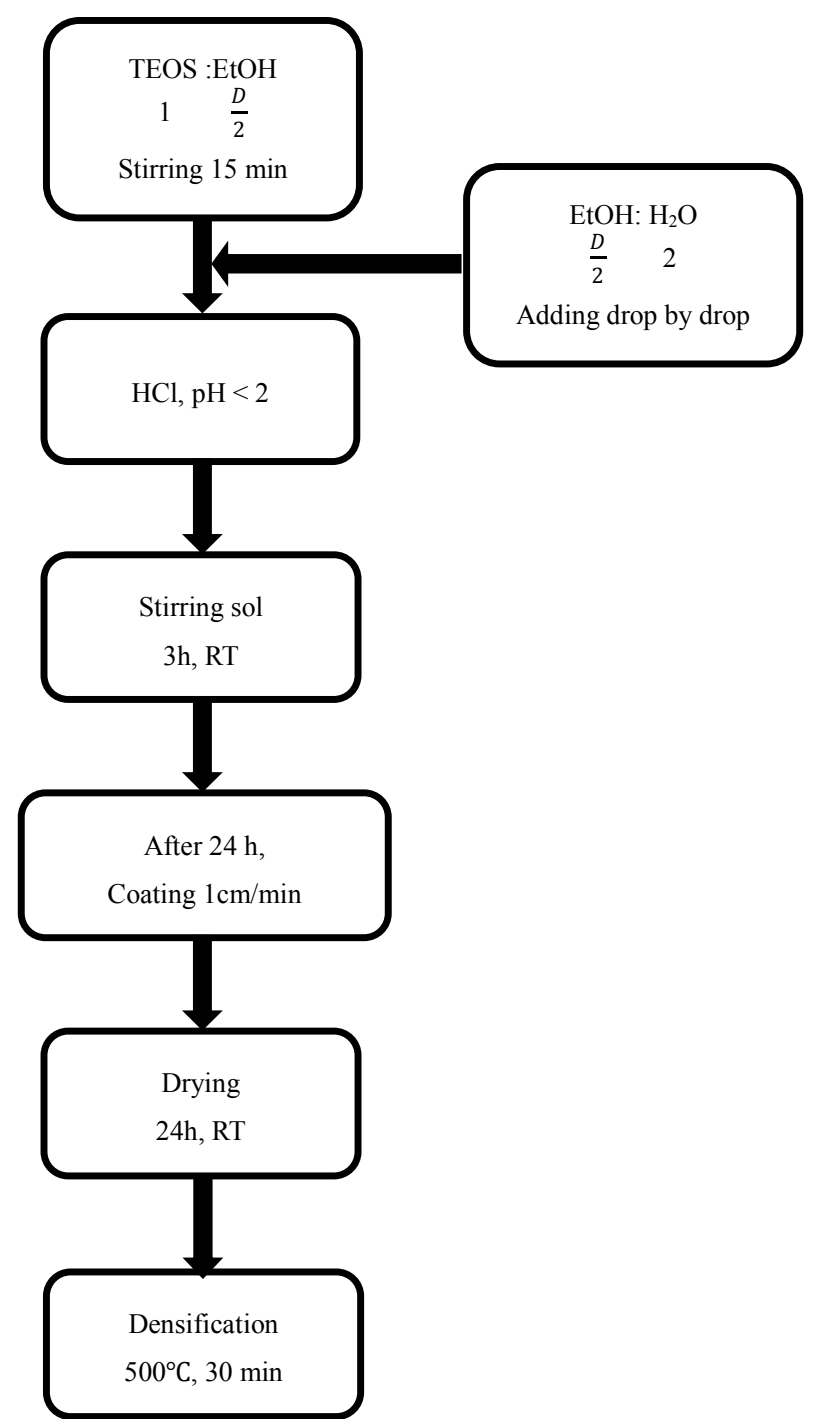

Figure 1. Flow chart of sol-gel processing for $\mathrm{SiO} 2$ films (The D factor indicate EtOH ratio molar) 


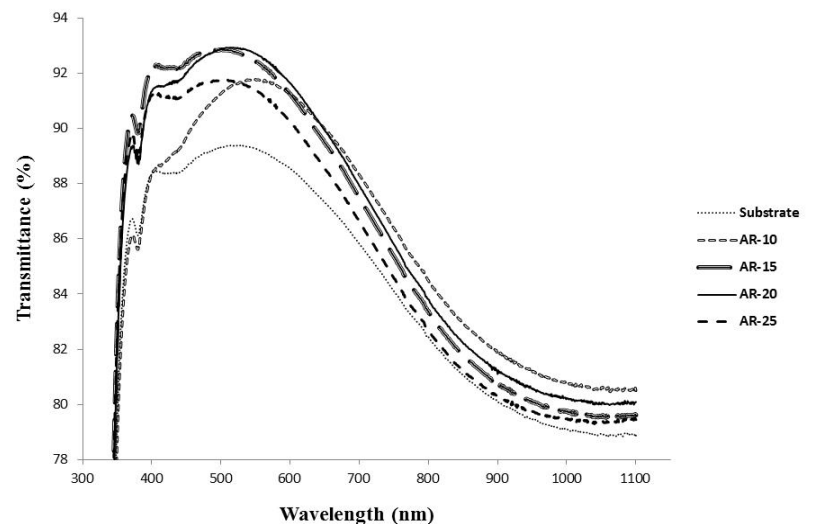

Figure 2. Optical transmittance spectra of coatings with different $D$ factor

Figure 2 also demonstrates that $\mathrm{D}$ ratio of 20 is a sufficient and optimum amount when using ethanol as solvent in the sol of glass, because it provides around $3.96 \%$ increment in the transmittance rate relative to the bare sample.

By increasing solvent content, sol became dilute, so this factor increases the total porosity after coating and densification phase. The more porosity on the coating, the less optical reflective index and consequently transmittance increased. It should be noted that porosity increment due to solvent content increasing may influence coating lifetime.

From these optical transmittance spectra (as shown in figure 2), average transmittance values have been calculated in two different intervals: visible $(400-800 \mathrm{~nm})$ and infrared $(800-1100 \mathrm{~nm})$, in order to quantify and evaluate the influence of $\mathrm{SiO}_{2}$ sol dilution on each spectral region. The average transmittance ratios $\left(\mathrm{T}_{1} / \mathrm{T}_{0}\right)$ of the $\mathrm{SiO}_{2}$ coated samples $\left(\mathrm{T}_{1}\right)$ and the uncoated glass substrate $\left(\mathrm{T}_{0}\right)$ are represented in Figure 3, both in the visible and infrared ranges, as a function of the precursor dilution (D) at the withdrawal velocity of $v=$ $1 \mathrm{~cm} / \mathrm{min}$.

At withdrawal velocity of $\mathrm{v}=1 \mathrm{~cm} / \mathrm{min}$, visible transmittance enhancement varies from 2.4 to $3.2 \%$ as the $\mathrm{D}$ ratio increase from 10 to 20 . Futhermore, increasing D ratio decrease the transmittance ratio due to sol diluteness because of increasing solvent and prosity.

As it can anticipated, in the infrared optical range, the transmittace ratio decreases relatively. Similar to visible, the $\mathrm{D}=20$ point is the best condition in the infrared rang. It has been proven that sol-gel $\mathrm{SiO}_{2}$ covers the substrate surface like a paste, smoothing its irregularities, thus resulting in a heterogeneous $\mathrm{SiO}_{2}$ thickness, higher over the valleys than over the protuberances of the substrate surface. In the present study the sol-gel coverage on the substrate increases as the $\mathrm{D}$ factor increases up to 20 . The observed increase in the visible range transmittance can be attributed to the $\mathrm{SiO}_{2}$ layer continuity on the glass substrate.

The micrograph of the surface of the $\mathrm{SiO}_{2}$ nanostructure film, as shown in figure 4 (a-c), contains submicron pores. Size of $\mathrm{SiO}_{2}$ grains can be estimated to be in the range of 20-40 nm.

As is clear, the more D factor, the more porosity and the less reflective index and the more optical transmittance.
With increasing $\mathrm{D}$, the sol concentration became more dilute so that by evaporation of solvent, the coating thickness and weight per $\mathrm{cm}^{2}$ lessen and therefore this process can lead to more porous coating. These results are compatible with what mentioned in Figure 2 \& 3.

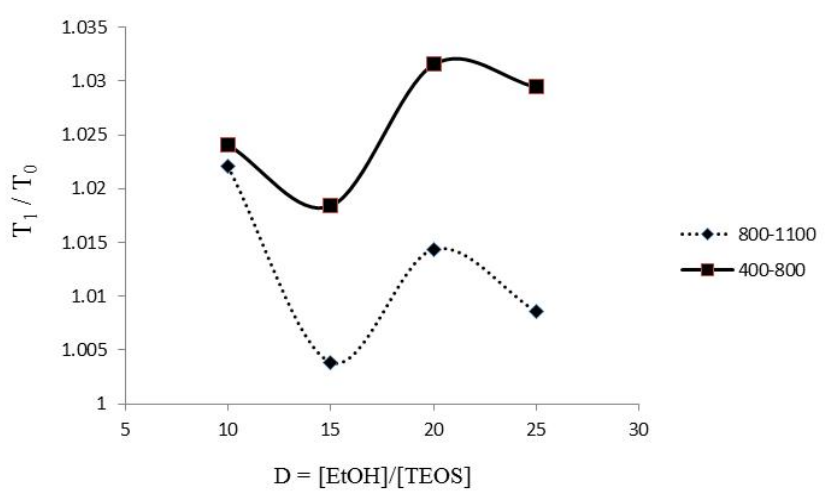

Figure 3. Transmittance ratio (verage of $\mathrm{T}_{1} / \mathrm{T}_{0}$ ) of the $\mathrm{SiO}_{2}$-coated samples, $T_{1}$, vs. the glass substrate, $T_{0}$, as a function of the precursor dilution, $D$
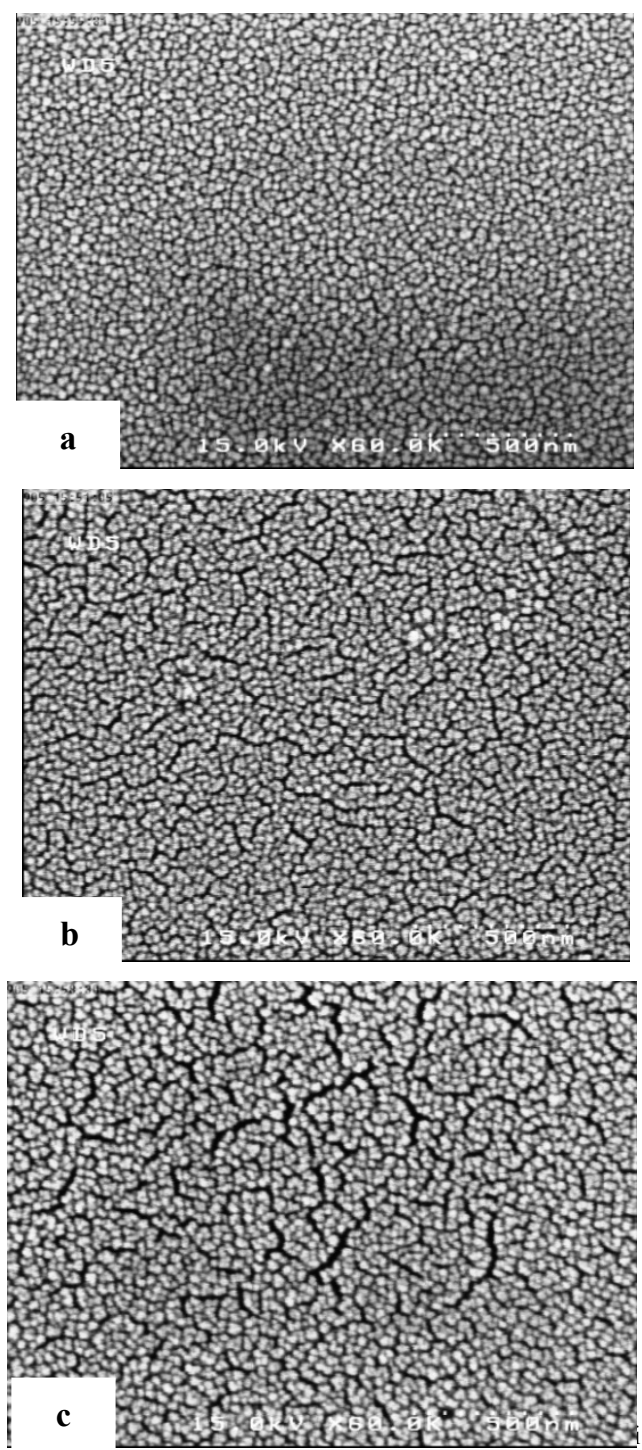

Figure 4. FE-SEM micrograph of surface $\mathrm{SiO}_{2}$ films, (a) $\mathrm{D}=10$, (b) $\mathrm{D}=15$, (c) $\mathrm{D}=20$ 


\section{Conclusions}

Uniform nanostructure $\mathrm{SiO}_{2}$ film was applied on glass substrate in this study with the least defects. The optical effects of microstructure of $\mathrm{SiO}_{2}$ sol-gel coatings on the glass substrate were investigated. This research proved that with increasing $\mathrm{D}$, the porosity and light transmittance increased. With increasing amount of $\mathrm{D}$, the optimum thickness decreases therefore the reflective index decreases and light transmittance increases. The best anti-reflective behavior showed the coatings obtained from $\mathrm{D}=20$ and the highest absolute transmittance (92.94\%) was achieved at approximately $500 \mathrm{~nm}$ wavelength therefore sol-gel $\mathrm{SiO}_{2}$ coatings on glass substrate can provide about 4 and $1.2 \%$ optical transmittance enhancement in the visible infrared range respectively.

\section{ACKNOWLEDGEMENTS}

This work was partially financially supported by supplying automotive parts company (SAPCO).

\section{REFERENCES}

[1] Chen, D., 2001, Anti-reflection (AR) coatings made by sol-gel processes: A review., Sol. Energy Mater. Sol. Cells 68, 313 .

[2] Nostell, P., Roos, A., Karlsson, B., 1999, Optical and mechanical properties of sol-gel antireflective films for solar energy applications., Thin Solid Films, 351, 170.

[3] Gombert, A., Glaubitt, W., Rose, K., 1998, Glazing with very high solar transmittance., Solar Energy, 62, 177.

[4] Alam, M.J., Cameron, D.C., 2001, Characterization of transparent conductive ITO thin films deposited on titanium dioxide film by a sol-gel process., Surface Coating Technology 142-144, 776 .

[5] Abe, K., Sanada, Y., Morimoto, T., 2001, Anti-Reflective Coatings for CRTs by Sol-Gel Process., Sol-Gel Sci. Technol., 22, 151-153.

[6] Wu, W.F., Chiou, B.S., 1997, Mechanical and optical properties of ITO films with anti-reflective and anti-wear coatings., Appl. Surf. Sci., 115, 96.
[7] Samuneva, B., Kabaivanova, L., Chernev, G., Djambaski, Kashchieva, E., et al. 2008, Sol-gel synthesis and structure of silica hybrid materials., Sol-Gel Sci. Technol., 48, 73-74.

[8] Morales, A., Dur'an, A., 1997, Sol-Gel Protection of Front Surface Silver and Aluminum Mirrors., Sol-Gel Sci. Technol., 8,451 .

[9] Yashan, G.R., Ermenko, A.M., Smirnova, N.P., Suzer, S., Ertas, G., et al., 2008, Morphology and Optical Properties of Thin Silica Films Contained Bimetallic Ag/Au Nanoparticles., Theoretical and Experimental Chemistry, 44, 356-357.

[10] Evans, P., English, T., Hammond, D., Pemble, M.E., Sheel, D.W., 2007, The role of $\mathrm{SiO}_{2}$ barrier layers in determining the structure andphotocatalytic activity of $\mathrm{TiO}_{2}$ films deposited on stainless steel., Applied Catalysis A: General, 321, 141.

[11] Jo, M.H., Hong, J.K., 1997, Application of $\mathrm{SiO}_{2}$ aerogel film with low dielectric constant to intermetaldielectrics., Thin Solid Films, 308-309, 490.

[12] Schumacher, J.O., Hebling, C., Warta, W., 1997, Analysis and design of a thin film silicon solar cell on an insulating substrate., Proceedings of the 14th European photovoltaic Solar Energy Conference, Barcelona/Spain, 1467-1469.

[13] Tuttle, J.R., Berens, T.A., Keane, J., et al., 1996, Investigations into alternative substrate, absorber and buffer layer processing for $\mathrm{Cu}(\mathrm{In}, \mathrm{Ga}) \mathrm{Se}_{2}$-based solar cells., 25th IEEE Photovoltaic Specialists Conference, Washington, DC, USA, 797-798.

[14] S.Hegedus, S., Albright, S., et al., 1997, Substrates, contacts and monolithic integration.,Prog. Photovoltaics, 5, 365.

[15] O“zsan, M.E., Johnson, D.R., et al., 1994,Characterisation of $\mathrm{CdS} / \mathrm{CdTe}$ Thin Film Solar Cells., 12th European Photovoltaic Solar Energy Conference, 1604-1605.

[16] Liu, B.T., Yeh, W.D., 2010, Colloids and Surfaces A: Physicochemical and Engineering Aspects Antireflective surface fabricated from colloidal silica nanoparticles, 365, 145-149.

[17] Mohallem, N.D.S., Aaegerter, M.A., 1988, Multilayer $\mathrm{SiO}_{2}$ and $\mathrm{TiO}_{2}$ coatings on glasses by the sol-gel process., Non-Crystalline Solids, 100, 526-530.

[18] Brinker, C.J., Scherer, G.W., 1990, Sol-Gel Science., Academic Press, New York.

[19] Guill'en, C., Morales, A., Herrero, J., 2000, Performance of sol-gel $\mathrm{SiO}_{2}$ coatings onto glass $/ \mathrm{SnO}_{2}$ superstrates., Surface and Coatings Technology, 132, 31-35. 